\title{
Transatlantica
}

Revue d'études américaines. American Studies Journal

\section{Fur : un portrait imaginaire de Diane Arbus}

Jean Kempf et Morgan Riou

\section{(2) OpenEdition}

\section{Journals}

Édition électronique

URL : http://journals.openedition.org/transatlantica/1321

DOI : 10.4000/transatlantica.1321

ISSN : 1765-2766

Éditeur

AFEA

\section{Référence électronique}

Jean Kempf et Morgan Riou, «Fur : un portrait imaginaire de Diane Arbus », Transatlantica [En ligne], 1। 2007, mis en ligne le 09 juillet 2007, consulté le 29 avril 2021. URL : http://journals.openedition.org/ transatlantica/1321; DOI : https://doi.org/10.4000/transatlantica.1321

Ce document a été généré automatiquement le 29 avril 2021.

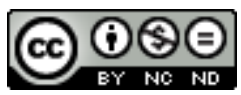

Transatlantica - Revue d'études américaines est mis à disposition selon les termes de la licence Creative Commons Attribution - Pas d'Utilisation Commerciale - Pas de Modification 4.0 International. 


\title{
Fur : un portrait imaginaire de Diane Arbus
}

\author{
Jean Kempf et Morgan Riou
}

1 Fur, film américain de Steven Shainberg, sorti en France début janvier 2007, propose un " portrait imaginaire de Diane Arbus », photographe américaine des années 1960, devenue célèbre par quelques-unes de ses images étranges et inquiétantes, telles celle du jeune garçon tenant une grenade dans Central Park ou celles des jumelles en habit du dimanche, mais surtout en raison de sa vie tourmentée et de son suicide en 1971 à l'âge de 48 ans. Le film de Shainberg (qui s'était fait connaître par The Secretary, une histoire de sado-masochisme) est inspiré, tout au moins pour sa partie «réaliste», de la biographie de Patricia Bosworth parue en $1984^{1}$.

2 Le réalisateur s'est appuyé sur cet ouvrage de Bosworth et un certain nombre de scènes du film sont en effet fidèles à ce que rapporte l'auteur de son personnage. Le film débute au moment où Diane Arbus, née Diane Nemerov, ${ }^{2}$ fille du proprétaire d'un grand magasin new-yorkais (Russek's), alors assistante de son mari (photographe de mode dans l'entreprise de son beau-père) commence à envisager pour elle-même une carrière de photographe. Il s'agit donc d'une période charnière de sa vie, celle où bascule un destin. Le film s'ouvre sur la réalisation du portrait « Retired man and his wife at home in a nudist camp one morning, N.J. 1963 ». Diane Arbus y apparaît donc, dès le début, comme la photographe de la différence, même si à l'époque le mot existe à peine. S'amorce ensuite un long flash-back, qui durera tout le film, jusqu'au retour à la scène finale en clôture. A travers ce parcours, Shainberg évoque le processus par lequel Diane Arbus est passée du rôle classique d'épouse et de mère des années quarante ou cinquante, à celui de femme émancipée des années soixante, s'aventurant dans les marges de la société, fascinée par l'interdit, le caché, les bas-fonds ou, comme le littéralise une scène du film, les égouts.

3 Tout cela ne suffit pas à faire de Fur un bon film, et même un film intéressant. Plusieurs raisons à cela : certaines purement cinématographiques, d'autres plus «structurelles ». Ce sont les motifs structurels de cet échec que nous souhaitons développer ici, car ils 
éclairent les problèmes que posent à la fois la photographie de Diane Arbus et l'idée même de biographie artistique.

4 Fur est le portrait d'une déviance, d'un décrochage du mainstream, sujet hollywoodien par excellence (le misfit étant probablement une figure du cinéma américain plus centrale que le All-American guy). Ici, il est question à la fois d'une transgression sociale et culturelle (la jeune mère de bonne famille, bourgeoise tendance nouveau-riche, s'encanaille chez les marginaux et, du coup, abandonne son rôle de femme au foyer) et d'une transgression psychologique, puisque quelque chose du rapport au réel se défait chez cette femme qui devient victime d'hallucinations. Tel est sans doute le prix à payer pour l'art ou le génie. C'est probablement cet aspect du film qui est le plus réussi, au moins au niveau de l'idée, si ce n'est de l'originalité. Afin de pouvoir scénariser efficacement ce moment de la vie de Diane Arbus où, de simple assistante de sa star de mari, elle devient photographe à part entière et par là même une femme "libérée " (prête à en assumer les risques), les concepteurs du film ont choisi l'unité de temps (le tout début de ses reportages "personnels »), de lieu (l'immeuble cossu où les Arbus viennent d'emménager) et la littéralisation de la métaphore de la rupture, en lui inventant de toutes pièces un étrange voisin du quatrième étage, s'éloignant ici radicalement de la biographie de Bosworth et créant ainsi, avec ce diable sécularisé, une sorte de remake de Rosemary's Baby. Le mystérieux voisin au masque de cuir, monstre de foire atteint d'une maladie dégénérative, l'hypertrichose, fait d'elle sa proie psychologique et sexuelle, qu'il entraîne dans le monde des ombres et des monstres, à travers un jeu de séduction morbide réciproque - mort et voyeurisme cohabitent et s'enchâssent - et de pathos - moins réciproque car si Arbus plaint ces êtres en marge, eux ne s'apitoient jamais sur leur sort ; bien au contraire ils le vivent entre enjouement hystérique et acceptation naturelle. Le problème est moins dans l'idée, somme toute acceptable bien que peu originale, que dans la réalisation qui, en littéralisant lourdement les métaphores, vire le plus souvent au kitsch, particulièrement sensible dans les choix « gothiques » des décors.

5 Se pose ensuite le problème de la représentation et mise en scène de la création. Fur s'inscrit dans une série de films sur des créateurs, peintre (Vincent, Leonard Nimoy, 1981) ou musicien (Music Lovers, Ken Russell, 1970), par exemple, qui tentent, au-delà de l'entreprise biographique qui sert de support visuel au film, de sonder l'insondable ou de cerner l'incernable, à savoir le processus créatif lui-même. L'intention est toujours de saisir chez l'aventurier - fût-il de l'art ou de l'esprit - ce moment décisif où, tout à coup, la matière est transfigurée : comment un stylo et une feuille de papier, de simples pigments et un morceau de toile, produisent soudain un artefact jamais vu, doté d'une vie autonome. C'est ici le thème de l'alchimie qui est visé, où advient de l'inouï dans le plus trivial des matériaux (la photographie en tant que dernier des beaux-arts étant en ce sens exemplaire). Mais ici le réalisateur ne se contente pas d'une représentation même modeste mais minutieuse des formes du travail. Dans le cas de la photographie, ce travail serait double : travail technique et matériel de l'artiste - comme dans le cas de la peinture - et travail corporel et psychologique entre l'artiste et son modèle - comme au théâtre. Il se joue en effet toujours, dans la photographie, un échange complexe - souvent physique - entre le monde et un regard incarné qui se fige, à un moment donné, dans une forme plastique pérenne. Ce processus original n'est pas sans rapport avec le cinéma. Celui-ci, en tant qu'art de narration (et donc de fiction), est à la fois fort éloigné de la photographie (qui penche vers le constat et n'a rien de narratif) et pourtant il partage avec elle la mise en scène de la corporéité d'un 
regard. De ce point de vue, Diane Arbus est l'artiste idéale, ou la victime parfaite si l'on s'en tient aux résultats, tant son œuvre est fondée sur la tension de corps s'affrontant au travers de l'appareil-photo dans un rite de pose à la fois simple (si on le compare à celui d'autres portraitistes) et tendu à l'extrême (en raison des situations et des sujets choisis).

Devant la difficulté extrême à mettre en scène et à donner à voir cette relation dans laquelle se joue la création et son mystère, Steven Shainberg espère que la métaphore pourra lui permettre de retrouver une théâtralisation qui lui échappe, d'où une focalisation sur la transgression de cette femme psychologiquement fragile, très belle et très inquiétante.

7 Travaillé de l'intérieur par cette volonté de connaître qui évidemment le détruit, le personnage de Diane a toute les composantes de l'artiste maudite. Freaks et poète sont faits pour se comprendre. Mais ici, il s'agit d'une femme dont tout porte à croire qu'elle est frustrée (bien sûr sexuellement d'abord) et que sa véritable libération, dans cette upper-middle class new-yorkaise du début des années 60 qui reste totalement hermétique aux bouleversements qui secouent l'Amérique, consiste, pour la belle, à aller vers la bête. On aura compris que c'est dans cette transgression (littéralement

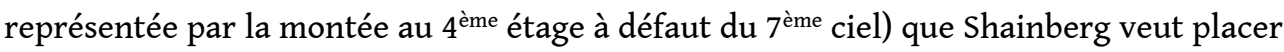
la vraie révolution sociale de l'époque et le terreau d'une conscience d'artiste. On reste un peu confondu devant de telles lourdeurs.

8 Pour en savoir un peu plus sur Diane Arbus, on ira plutôt voir du côté de la biographie qu'il y a 21 ans (1986) Patrick Roegiers consacrait à la photographe sous le titre «Le rêve du naufrage $"{ }^{3}$ Travaillant à partir des matériaux limités disponibles, Roegiers propose un intéressant travail de modelage du personnage. Certes, son livre est également fondé sur une mystique romantique du créateur et sur un rapprochement (indirect, à travers des citations) avec Kafka qui n'est pas nécessairement éclairant (Roegiers lui-même se pense comme un écrivain dans la lignée de Kafka, et les phénomènes d'auto-identification avec lui ne sont pas absents). L'un des problèmes majeurs concernant les travaux sur Arbus est qu'ils puisent sans relâche dans un fond de lecture d'inspiration psychanalytique: psycho-critique projective des images et psycho-exégèse de la biographie. Ce qui n'est ni surprenant ni totalement dénué d'intérêt.

9 Peu surprenant car jusqu'à aujourd'hui le contrôle total de la documentation et de l'accès aux archives qu'exerce Doon Arbus (pour des motifs qui posent le problème de la postérité des artistes et de la place des ayant-droits) a empêché un certain nombre de travaux historiques sérieux et limité l'investigation aux aspects extérieurs et à des fragments de l'œuvre. Heureusement, depuis la fameuse monographie Aperture, trois volumes sont venus compléter le corpus publié, sans totalement résoudre le problème.

Peu surprenant non plus car, bien sûr, certaines images, la vie même de Diane Arbus et son suicide, invitent « naturellement » à la dérive de l'interprétation. Son insistance sur le «secret» a également contribué à épaissir le mystère (évoqué dans cette merveilleuse citation en forme de pirouette: «A photograph is a secret about a secret »).

11 Aujourd'hui, en revanche, cette œuvre exige d'autres interprétations. Dans les années 1970 et 1980, la lecture généralement admise était qu'Arbus était une sorte de hapax de la photographie américaine, presque sans ancêtre et en tout cas sans descendance. Outre qu'il était en effet un peu tôt pour porter ce genre de jugement, il nous semble 
que cette remarque révèle bien la manière dont a évolué la critique photographique. Tout d'abord, il semble qu'on ait trop vite fait l'économie du genre "portrait» dans lequel Arbus s'inscrit nettement et qui constitue l'un des centres trop peu explorés de la photographie américaine. A l'époque, attachés à la forme ou au message (fût-il plus psychologique que social ou historique), les critiques pensaient assez peu en termes de pratiques. Pour le dire autrement, la sémiologie et l'esthétique dominaient l'interprétation, et on se préoccupait peu de la manière dont formes, processus, usages s'intégraient dans une culture globale, qu'ils participaient à transformer. Or, ce qui finalement reste subversif chez Arbus, est moins la déviance montrée que le retournement radical du portrait et son investissement monomaniaque. Ce que l'on connaissait déjà bien dans les années 70 , à travers les témoignages et images disponibles, et que Revelations a démontré de manière claire, est à quel point cette obsession à construire une œuvre n'était que le pendant " artistique » d'une obsession phénoménologique, et même métaphysique, celle de la collection. On sait en effet combien la thésaurisation que permet (et appelle même) la photographie comme dispositif de prélèvement souple et maniable du réel a joué dans son invention et son développement. On en trouve très tôt (1839) des manifestations dans l'usage scientifique, architectural, voire pictural et muséal de l'image photographique. Mais c'est dans la photographie d'exploration depuis la seconde moitié du XIX ${ }^{e}$ siècle et dans le développement de la pratique du documentaire, en particulier social, que le concept de collection s'est pleinement développé, singulièrement d'ailleurs aux Etats-Unis. Et dans/de cette histoire, le portrait vu aussi comme mode de collection des êtres, surtout lorsqu'il est ethnographique ou social, a écrit un chapitre particulièrement important. La pratique collectionneuse de Diane Arbus, qu'il faut aujourd'hui reprendre à nouveaux frais, constituerait une grille de lecture puissante qui la réinscrirait de manière magistrale dans l'histoire des pratiques de l'image aux Etats-Unis ${ }^{4}$.

\section{BIBLIOGRAPHIE}

Bosworth, Patricia. Diane Arbus : a Biography (New York : Knopf, 1984).

Diane Arbus : Revelations. New York : Random House, 2003.

Diane Arbus. Millerton, N.Y. : Aperture, 1972.

Lee , Anthony W. et John Pultz. Diane Arbus : Family Albums. New Haven, CT : Yale University Press, 2003.

Magazine Work. Millerton, N.Y. : Aperture, 1984.

Roegiers, Patrick. Diane Arbus ou le Rêve du naufrage (Paris : Chêne, 1985).

Untitled. New York, N.Y. : Aperture, 1995. 


\section{NOTES}

1. Patricia Bosworth, Diane Arbus : a Biography (New York : Knopf, 1984).

2. Son frère est le poète Howard Nemerov.

3. Patrick Roegiers, Diane Arbus ou le Rêve du naufrage (Paris : Chêne, 1985) republié parPerrin en 2006.

4. Patrick Roegiers, dans son introduction à la réimpression en 2006 de son essai de 1985 tente fort justement cette réinscription dans l'histoire de la photographie. Il place Arbus en compagnie de Ralph-Eugene Meatyard son contemporain, et voit en elle l'inspiratrice d'une Nan Goldin ou d'une Cindy Sherman, voire d'un Mapplethorpe. Sans nier ces liens, nous pensons que ces généalogies sont réductrices car fondées sur des formes plus que des pratiques (profondes). Cela le conduit à une conclusion séduisante mais probablement erronée, à savoir que cette photographie-là «ne serait plus admise ni même envisageable, pour ne pas dire, tout bonnement impensable » dans l'Amérique d'aujourd'hui. C'est opérer une lecture rapide (et assez française, même si Roegiers est belge) de la prétendue "normalisation » américaine, et surtout c'est nier que la subversion se déplace toujours, y compris dans des sociétés prétendument "libres », et donc viser chez Arbus, comme nous le disions, plus un signifiant qu'un signifié.

\section{AUTEURS}

\section{JEAN KEMPF}

Université Lumière - Lyon 2

\section{MORGAN RIOU}

Université de Brest 